دراسة وتحليل العمليات الرياضـية للمنطق المضــبب

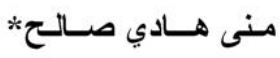

تاريخ قبول النشر 28 / /2009

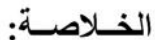

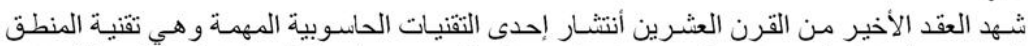

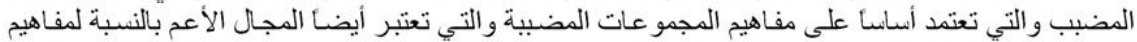

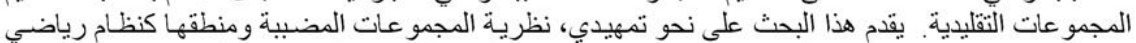

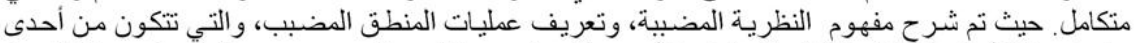
عشرة عملية أساسية. بالإضافة الى العمليات الأخرى التي يتضمنها الجبر المضبب. يعد هذا البحث مدخلا لإسناد بحوث أخرى في هذا الموضوع ع المهم والحيوي.

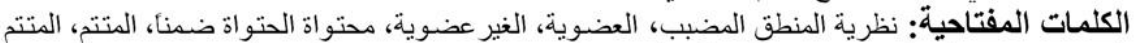

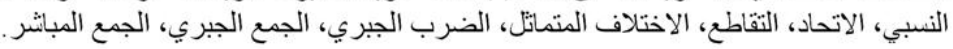

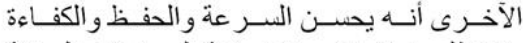

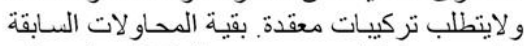

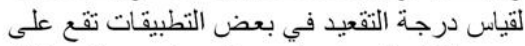

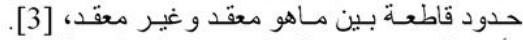

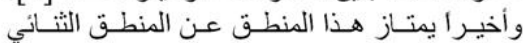

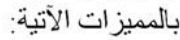

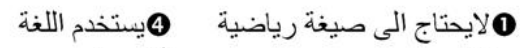
الطبيعية.

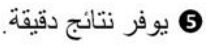
2 سنهل في التعامل والتعمل بشكل جيد مع بقية التقنيات

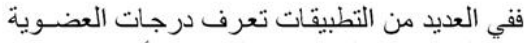

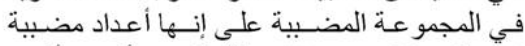
(Fuzzy Numbers)

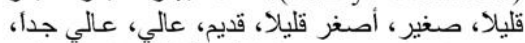

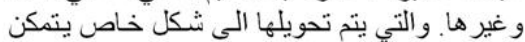

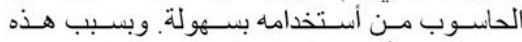

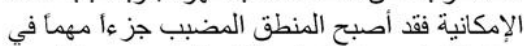

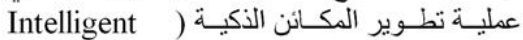
$[3,2]$ (Machines

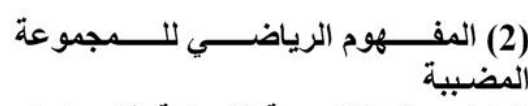

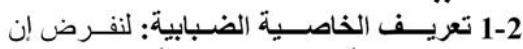

Space ) هي فضـاء أو مجمـو عة من الأشياء (X)

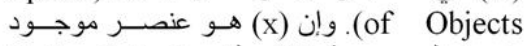

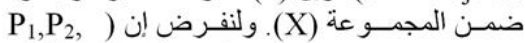

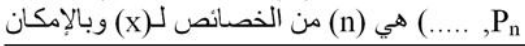

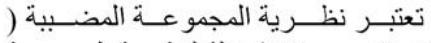
شـاملة لنظـرية المجموعة (Fuzzy Set Theory التقليدية (Abstract Set Theory) ذات الحدود

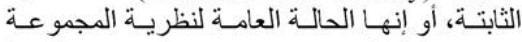

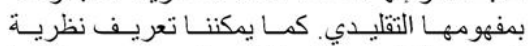

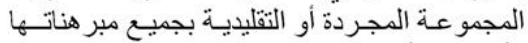

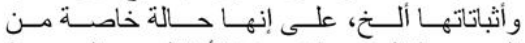

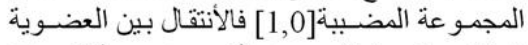

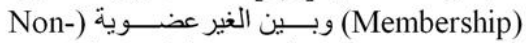

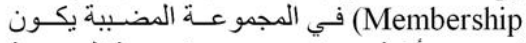

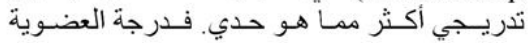
تتحدد بواسطة عدد العة فية (Grade Of Membership) معين يقع ضمن الفترة المغلقة [1,0] أي يقع بين بين

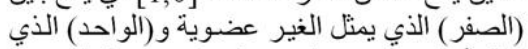

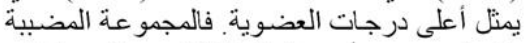

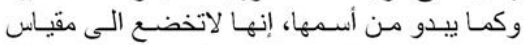

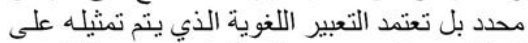

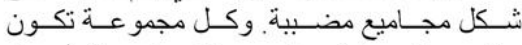

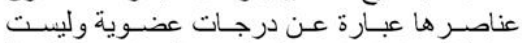

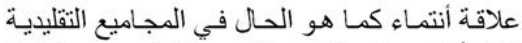

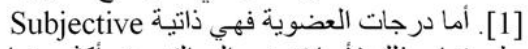
بطبيعتها وذللك لأنها تخضع التى التعريف أكثر منها

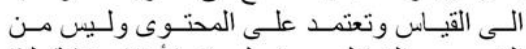

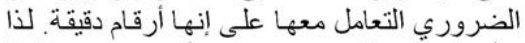

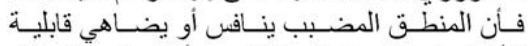
الأنسان في القدرة على التفكيير وأستعمال معلو القوات

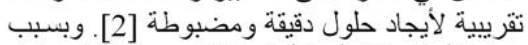

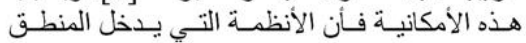

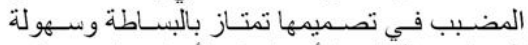

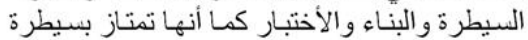
مرنة وناعمة مقارنة بالأنظمة التقليدية. من مميز اتهـ 


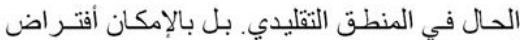

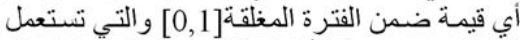
للإيعاز عن درجة الأنتماء التي يتم تمثيلهاً باستعمال تلتصل

المتغير ات اللفظية [5] درجة النتاء

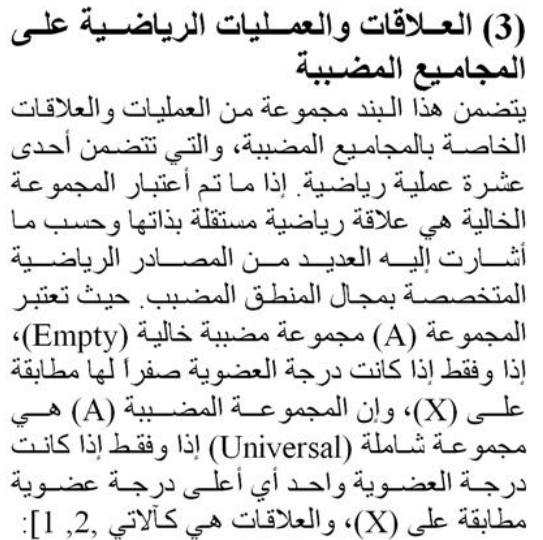

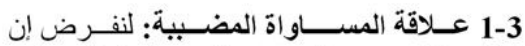

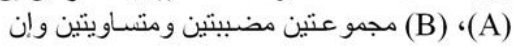

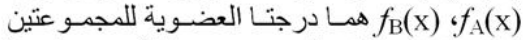

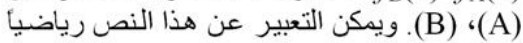

$\mathrm{A}=\mathrm{B} \quad$ iff $\quad f_{\mathrm{A}}(\mathrm{x})=f_{\mathrm{B}}(\mathrm{x})$

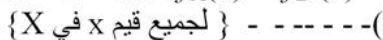

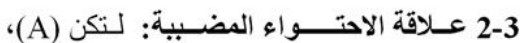

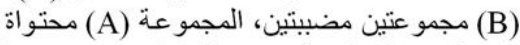

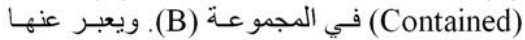
رياضيا $(\mathrm{A} \subseteq \mathrm{B}$

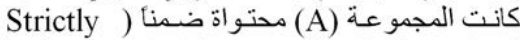

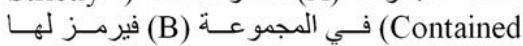

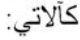

$$
\mathrm{A} \subset \mathrm{B} \text { iff } f_{\mathrm{A}}<f_{B} \ldots-(5)
$$

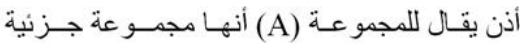
A $\subseteq$ من المجمو عة (B) (إذا كانت (Subset) (Proper Subset) ويقال لها جزئية مناسبة (B) إذا كانت (BᄃB)
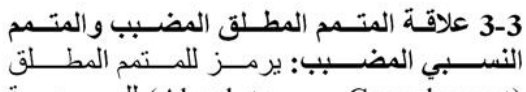
للمجموعـــا (Absolute Complement)

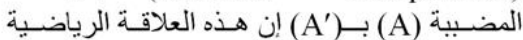
تستخدم بشكل واسـع في معظم التطبيقات العملية الترانية
أعتبار هـا (n) من المتغير ات حيث يرمز بـالحرف

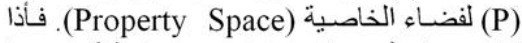

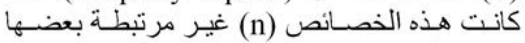

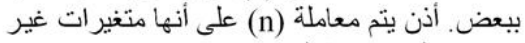

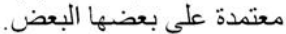

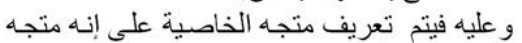

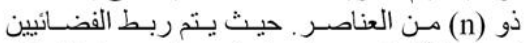

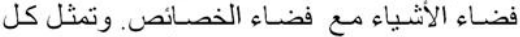

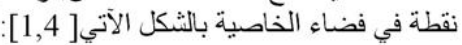
$\mathrm{X}=\left(\mathrm{P}_{1}, \mathrm{P}_{2}, \ldots, \mathrm{P}_{\mathrm{n}}\right)$ - . - . - (1) الآن لنصف المجموعة المضبية (A) في الفضـاء

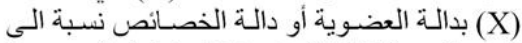

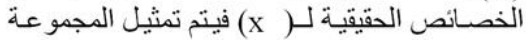

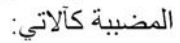

$f_{\mathrm{A}}\left(\mathrm{x}=\left(\mathrm{P}_{1}, \mathrm{P}_{2}, \ldots ., \mathrm{P}_{\mathrm{n}}\right)\right)$ - - - (2)

و هذه هي علاقة الترابط الدالي على فضاء الخاصية

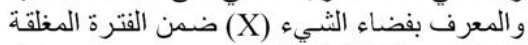
$f_{\mathrm{A}}\left(\mathrm{x}=\left(\mathrm{P}_{1}, \mathrm{P}_{2}, \ldots . . .\right.\right.$, .

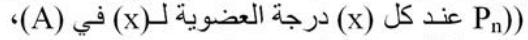

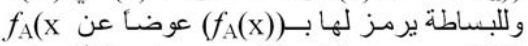
= ومن هذا التعريف

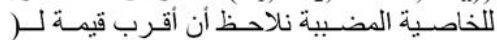

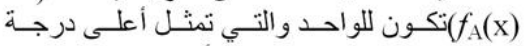

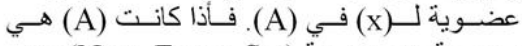
مجمو عة غير مضبية (Non- Fuzzy Set) وهي (A) وهي

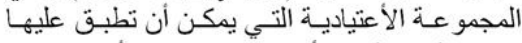

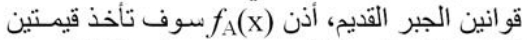

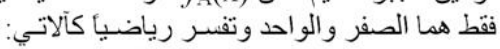

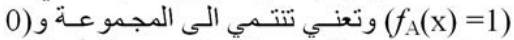

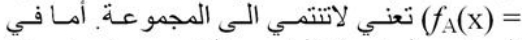

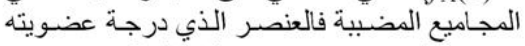

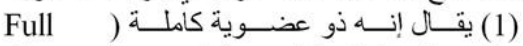
(Membership

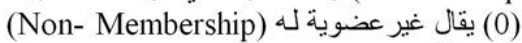

2-2 تعريـف المجموعـة المضبية: مـن التعـاريف

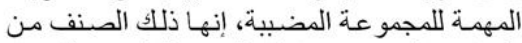
المجاميع التي تسمح بإمكانية تجزئة العضوية التئة فيها.

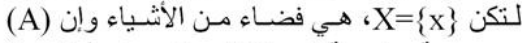

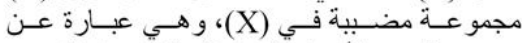
مجمو عـة مـن الأزواج المرنبـة والتــي يـتم التعبيـر

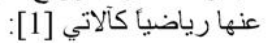
$\mathrm{A}=\left\{\mathrm{x}, \mu_{\mathrm{A}}(\mathrm{x})\right\} \quad \mathrm{x} \in \mathrm{X} \quad$ - - - - (3) حيث ان (ر) تمثل دالة درجة العضوية.

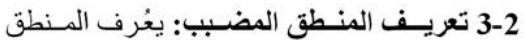

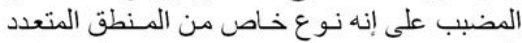

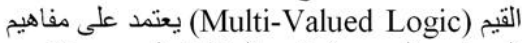

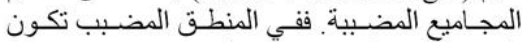
القيمة الحقيقية لمتغير ما، لاتأخذ قيمتين فقط كما هو المن 
بـالرمز (A+B) ويكـون نـاتج العمليــة مجموعـة

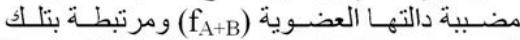
المجمو عتين (A, B)، يكتب ناتج العملية الرياضية

$f_{\mathrm{A}+\mathrm{B}}=f_{\mathrm{B}}+f_{\mathrm{A}}$

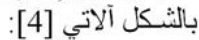

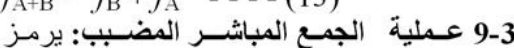

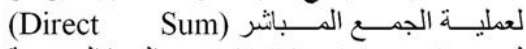

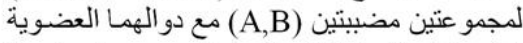

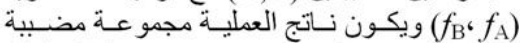

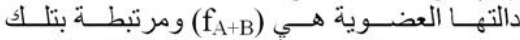

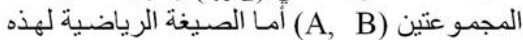
العملية قتكتب بالثكل آلاتي:

$f_{\mathrm{A} \oplus \mathrm{B}}=f_{\mathrm{A}+\mathrm{B}}-f_{\mathrm{AB}}--$ - (14)

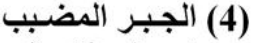

1-4 تعزيـف: ليكن الجبر المضبب عبارة عن نظام

منمثل بـآلاتي: خ

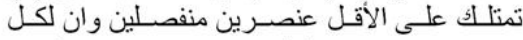

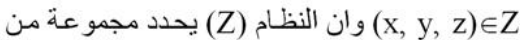

المبر هنات والمبينة في الجدول رقم (1) [1,4)

\begin{tabular}{|c|c|c|}
\hline & عمليات الضـات الربي & جدول رقمتم (1) \\
\hline$(x+x)=x$ & $(x . x)=x$ & النظظئley) \\
\hline$(x+y)=(y+x)$ & $(\mathrm{x} \cdot \mathrm{y})=(\mathrm{y} \cdot \mathrm{x})$ & الآت (Commutativity) \\
\hline $\begin{array}{l}(x+y)+z= \\
x+(y+z)\end{array}$ & $(\mathrm{xy}) \cdot \mathrm{z}=\mathrm{x} \cdot(\mathrm{y} z))$ & التجنمي \\
\hline$x+(x \cdot y)=x$ & $x \cdot(x+y)=x$ & الأتصنس (Absorption) \\
\hline $\begin{array}{l}x+(y \cdot z)=(x+ \\
y) \cdot(x+z)\end{array}$ & $\begin{array}{l}\mathrm{x} \cdot(\mathrm{y}+\mathrm{z})= \\
(\mathrm{x} \cdot \mathrm{y})+(\mathrm{x} \cdot \mathrm{z})\end{array}$ & التوزيسيس (Distributivity) \\
\hline
\end{tabular}

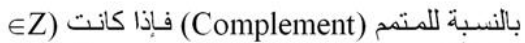

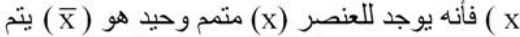
التعبير عنه كما في الجدول رقم (2) المبيد لمن ادناه

\begin{tabular}{|c|c|}
\hline \multicolumn{2}{|c|}{ جدول (2): أنــــواع المتممـات } \\
\hline نـوع المتمـم & التعليق \\
\hline$\overline{\mathrm{x}} \in \mathrm{z} \quad$ and $\quad \overline{\overline{\mathrm{x}}}=\mathrm{x}$ & المنتم المضاعف \\
\hline$\left(\mathrm{x}+\mathrm{e}^{+}\right)=\left(\mathrm{e}^{+}+\mathrm{x}\right)=\mathrm{x}$ & 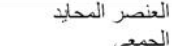 \\
\hline$\left(x \cdot e^{*}\right)=\left(e^{*} \cdot x\right)=x$ & الضربي المنصر المحايد \\
\hline$\overline{(x+y)}=(\bar{x} * \bar{y})$ & قو انين ديموركن \\
\hline$\overline{(x * y)}=(\bar{x}+\bar{y})$ & قو انين ديموركن \\
\hline $0+x=x ; 0 . x=0$ & الأصضغرغ المشنُرك \\
\hline $1+\mathrm{x}=1 ; 1 . \mathrm{x}=\mathrm{x}$ & 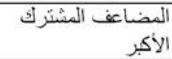 \\
\hline$(x \cdot \bar{x})+(y+\bar{y})=y+\bar{y}$ & $\begin{array}{r}\text { قو انين كلين's Laws) } \\
\text { (Kleene's }\end{array}$ \\
\hline$(x \cdot \bar{x}) \cdot(y+\bar{y})=x \cdot \bar{x}$ & $\begin{array}{r}\text { قو انين كلين } \\
\text { (Kleene's Laws) }\end{array}$ \\
\hline
\end{tabular}

كالمنطق المضبب وذلك لنسهولتها وتعرف رياضياً

$\bar{f}_{A}=1-f_{A}$

وإذا كانت كل من (A) ، (B) (B) مجمو عتين مضبتين

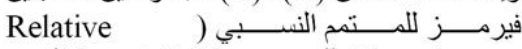
(Complement المجمو عة (B) - (B) - (B) وتعرف رياضياً هذه

$f_{B-A}=f_{B}-f_{A}$

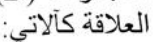

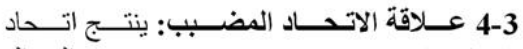

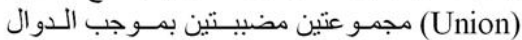
العضــية جديدة هي (C) )، وتكتب العـلاقة الرياضية كآلاتي:

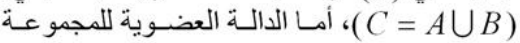

الجديدة ممكن ان تكتب بالثـكل آلاتي [5]

$f_{\mathrm{C}}(\mathrm{x})=\operatorname{Max}\left[f_{\mathrm{A}}(\mathrm{x}), f_{\mathrm{B}}(\mathrm{x})\right] \mathrm{x} \in \mathrm{X}$

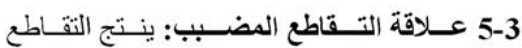

(A,B) بين مجمو عتين مضبيتين (Intersection)

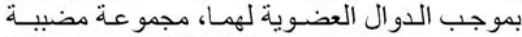

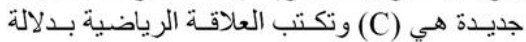

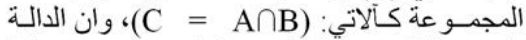

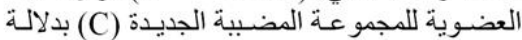
دوال العضوية للمجمو عتين (A,B) ممكن ان يعبر

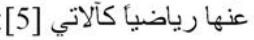
$f_{\mathrm{C}}(\mathrm{x})=\operatorname{Min}\left[f_{\mathrm{A}}(\mathrm{x}), f_{\mathrm{B}}(\mathrm{x})\right] \mathrm{x} \in \mathrm{X}$ - - - (9)

6-3 الاختـلاف المـتناظر المضــبب: تمثل عمليـة

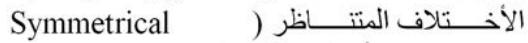
Boolean أو الجمـع البوليـاني (Difference

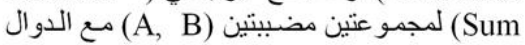
العضوية لكل منهما (f/ $f_{\mathrm{A}} \Delta f_{\mathrm{B}} \quad-\mathrm{y}_{\mathrm{B}}$ - (10)

أمـانـاتج العمليـة فهو مجمو عـة مضبية حيث دالـة العضوية للعملية مرتبطة بتلك المجمو عتين وكالآتي

$f_{\mathrm{A} \Delta \mathrm{B}}=\left|f_{\mathrm{A}}-f_{\mathrm{B}}\right|---(11)$

7-3 عــلية الضــرب الجبـري المضــبب: يرمـز

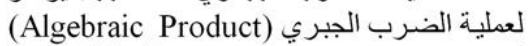

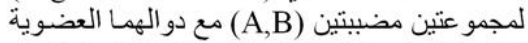

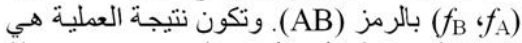

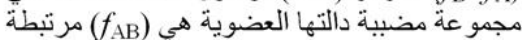

$\left.f_{\mathrm{AB}}=f_{\mathrm{B}} \cdot f_{\mathrm{A}}-\ldots-12\right)$

[4] بتلك المجمو عتين (AB)

8-3 عــلية الجمــع الجبــري المضــبب: يرهـز

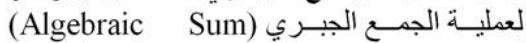
لمجمو عتين مضببتين (A, B) مع دو الهما العضوية 
(X) -2 هو متغير مضبب وكذلك (X) - (X)

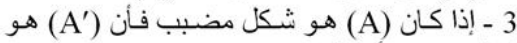
شكل مضبب أيضأ.

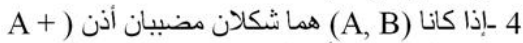
(BB) وكذلك (AB ) أنشكال مضبية. أذن الأثكال المضبية هي تلك المذكورة أعلاه من

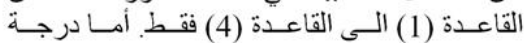

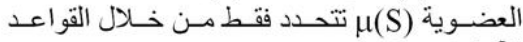
الآتية [6, ]

$\begin{array}{lll}1 & \mu(S)=0 & \text { if } S=0 \\ 2 & \mu(S)=1 & \text { if } S=1 \\ 3 & \mu(S)=\mu\left(x_{i}\right) & \text { if } S=x_{i} \\ 4 & \mu(S)=1-\mu(S) & \text { if } S=\bar{A} \\ 5 & \mu(S)=\operatorname{Min}\left[\mu_{A}(S), \mu_{B}(S)\right] & \text { if } S=A \cap B \\ 6 & \mu(S)=\operatorname{Max}\left[\mu_{A}(S), \mu_{B}(S)\right] & \text { if } S=A \text { U }\end{array}$

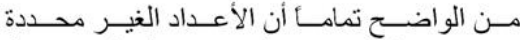

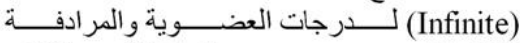
اللمتغيرات يوجد عدد محدود (Finite) من الثنائي

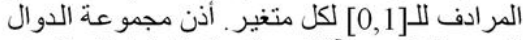

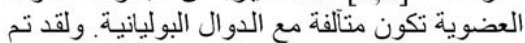

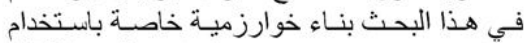
برنّامج (Visual Basic) وكما مبينة في الثكل رقم (1) تتعامل مع العلاقات الرياضية و الآعمليات

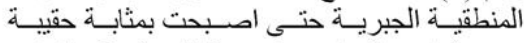
برمجية تخدم المهتمين في هذا المجال كمئ كات تعتبر حقيبة تعليمية للمبتدئين في مجال المنطق المضين المبال

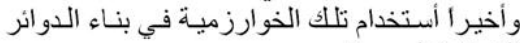
المنطقبة المضبية
من الواضـح أن النظلـام هو ذات توزيع شُبكي مـع النع

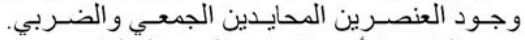

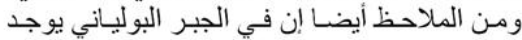

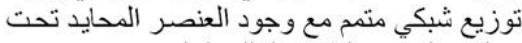

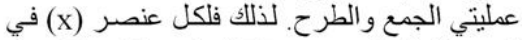
الجبـر البوليـاني يوجـد (x)

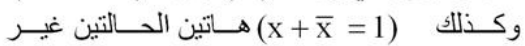

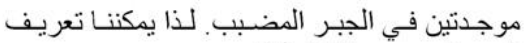
الجبر المضبب بالنظام آلاتي:

$Z=\langle[0,1],+, *,-\rangle-\cdots(15)$

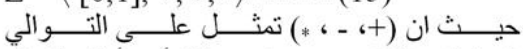
(Complement, Min, Max)

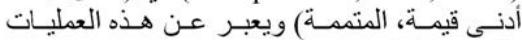

x = 1- x $\quad \forall x \in[0,1]$ - - -(16)

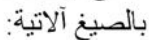

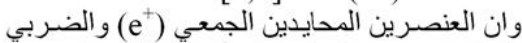

(e)

الجبر المضبب يتم تحديد هذان العنصران كآلاتي:

x. $0=0$ - ... (17)

$\mathrm{x}+0=\mathrm{x}$

$\mathrm{x} \cdot 1=1$

$\mathrm{x}+1=\mathrm{x} \quad \ldots \ldots(20)$

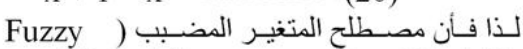
(Variable

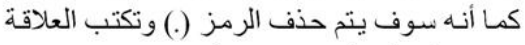

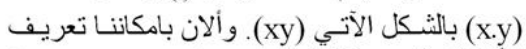
الأثكال المضببة المتولدة من ونسترجع القو اعد الأساسية الآتية: 1- الأرقام الغير متناهية التي تقع ضمن [0,1

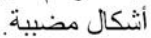

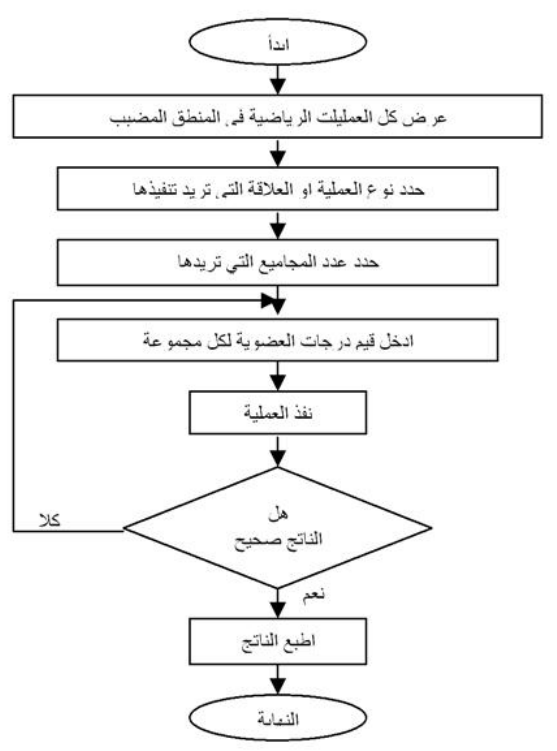

(5) (5ـــة الأمـبيقية

شكل (1): مخطط انسيابي يوضع خطو2 وتفيذ العمليكت الرياضية في المنطق المضبب 


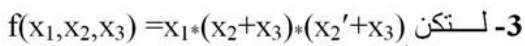

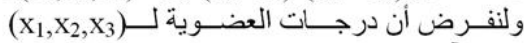

$=0.4$ ، $\mu\left(\mathrm{x}_{2}\right)=0.7$ ، $\mu\left(\mathrm{x}_{3}\right)=0.6$ كآلاتني

$f\left(\mathrm{x}_{1}, \mathrm{x}_{2},\left\{\mu\left(\mathrm{x}_{1}\right), \mu\left(\mathrm{x}_{2}+\mathrm{x}_{3}\right), \mu\left(\mathrm{x}_{2}{ }^{\prime}+\mathrm{x}_{3}\right)\right\}\right.$

$\left.\left.\mathrm{x}_{3}\right)\right]=\operatorname{Min}$

$=\operatorname{Min}\left\{\mu\left(\mathrm{x}_{1}\right), \operatorname{Max}\left\{\mu\left(\mathrm{x}_{2}{ }^{\prime}\right), \mu\left(\mathrm{x}_{3}\right)\right\}\right\}$

$\operatorname{Max}\left\{\mu\left(\mathrm{x}_{2}\right), \mu\left(\mathrm{x}_{3}\right)\right\}$,

$=\operatorname{Min}\{0.4$, Max $\{0.7,0.6\}$, Max $\{1-$

$\left.\left.\left\{\mu\left(\mathrm{x}_{2}\right), \mu\left(\mathrm{x}_{3}\right)\right\}\right\}\right\}$

$=\operatorname{Min}\{0.4,0.7, \operatorname{Max}\{1-0.7,0.6\}\}$

$=\operatorname{Min}\{0.4,0.7,0.6\}=0.4$

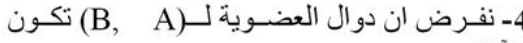

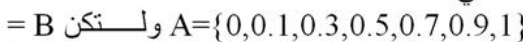


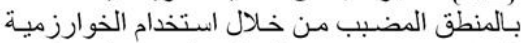

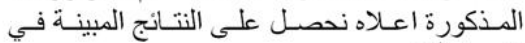

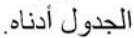

جدول(3):نتائج المسألة اعلاه.

\begin{tabular}{|c|c|c|c|c|c|}
\hline $\mathrm{A}+\mathrm{B}$ & $\mathrm{A}+\overline{\mathrm{A}} \mathrm{B}$ & $\overline{\mathrm{A}} \cdot \mathrm{B}$ & $\overline{\mathrm{A}}$ & $\mathrm{B}$ & $\mathrm{A}$ \\
\hline 0.2 & 0.2 & 0.2 & 1 & 0.2 & 0 \\
\hline 0.2 & 0.2 & 0.2 & 0.9 & 0.2 & 0.1 \\
\hline 0.3 & 0.3 & 0.2 & 0.7 & 0.2 & 0.3 \\
\hline 0.5 & 0.5 & 0.2 & 0.5 & 0.2 & 0.5 \\
\hline 0.7 & 0.7 & 0.2 & 0.3 & 0.2 & 0.7 \\
\hline 0.9 & 0.9 & 0.1 & 0.1 & 0.2 & 0.9 \\
\hline 1 & 1 & 0 & 0 & 0.2 & 1 \\
\hline
\end{tabular}

(6) المتـغيرات اللفظـية وأهمـيتها في المنطق المضـبب المغير

منذ سنين طويلة ولغاية الآن بقي مجال فهم كيفية

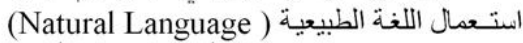
في التطبيقات المختلفة من الأمور الصـبة المبة أن لم

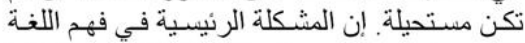

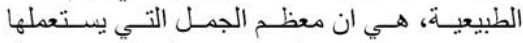

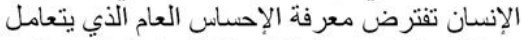

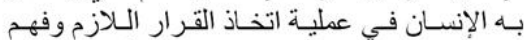

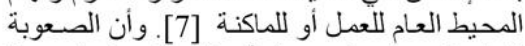

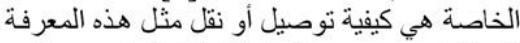

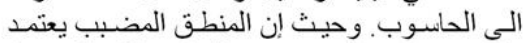

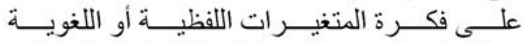
(Linguistic Variables) اللغويـة التي بألامكـان تمثيلهـا على شـكل مجـاميع

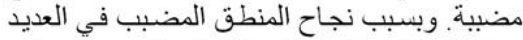
من التطبيقات والمجالات العلمية وكمـا موضِ فيح في العيد

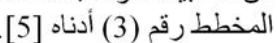

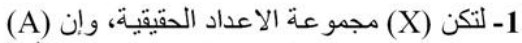

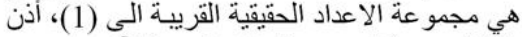
دالةّ العضوية للمجمو عة (A) تكون كالآتي:

$f_{A}(x)=\left[1+(x-1)^{2}\right]^{-1} \quad \mathrm{x} \in \mathrm{X}$

ولتكن (B) هي مجموعة الاعداد الحقيقية المغلقة الى (2) وأن دالة العضوية للمجمو عة (B) معرفة الأنة بالثكل الآتي: - ان

$f_{B}(x)=\left[1+(x-2)^{2}\right]^{-1} \quad \mathrm{x} \in \mathrm{X}$

فان اتحساد المجمو عتين (A,B) يعبر عنه رياضياً $f_{\mathrm{A} \cup \mathrm{B}}(\mathrm{x})=\operatorname{Max}\left[f_{\mathrm{A}}(\mathrm{x}), f_{\mathrm{B}}(\mathrm{x})\right]$

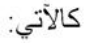

$f_{\mathrm{AUB}}(\mathrm{x})=\left[1+(\mathrm{x}-1)^{2}\right]^{-1} \quad \mathrm{x} \leq 1.5 \cdots-(21)$

$f_{\mathrm{AUB}}(\mathrm{x})=\left[1+(\mathrm{x}-2)^{2}\right]^{-1} \quad \mathrm{x} \geq 1.5$

x ) يكون تقاطعهما عند نقطة المنحنين

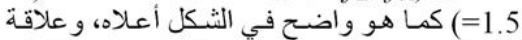

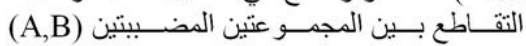
يعبر عنها :$f_{\mathrm{A} \cap \mathrm{B}}(\mathrm{x})=\operatorname{Min}\left[f_{\mathrm{A}}(\mathrm{x}), f_{\mathrm{B}}(\mathrm{x})\right]$

$f_{\mathrm{A} \cap \mathrm{B}}(\mathrm{x})=\left[1+(\mathrm{x}-1)^{2}\right]^{-1} \quad \mathrm{x} \leq 1.5-\cdots-\cdot-(23$

$f_{\mathrm{A \cap B}}(\mathrm{x})=\left[1+(\mathrm{x}-2)^{2}\right]^{-1} \quad \mathrm{x} \geq 1.5$--.----(24

من المعادلات أعلاه يتضح إن اتحساد المجمو عتين

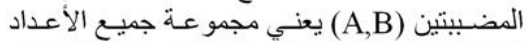
التي تكون مغلقة الى (1) والى (2) ولى ونف ونف الأستنتاج

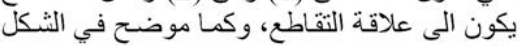

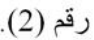

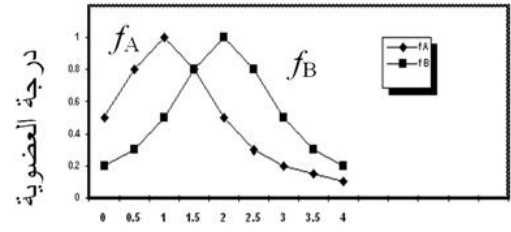

التوزيع الثامل (Universe of Discourse)

2- إذا كانت (X) مجمو عة جميع الأرقام الحقيقية الأكبر من واحد، وكانت (A) هي مجموعة الأن الأرقام

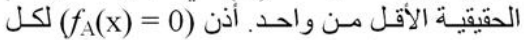

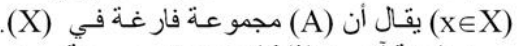

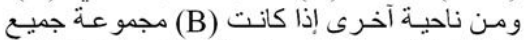

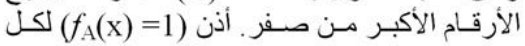
يقال حينئذ إن (B,X) هي مجمو عة شاملة في أني (x)

(X)

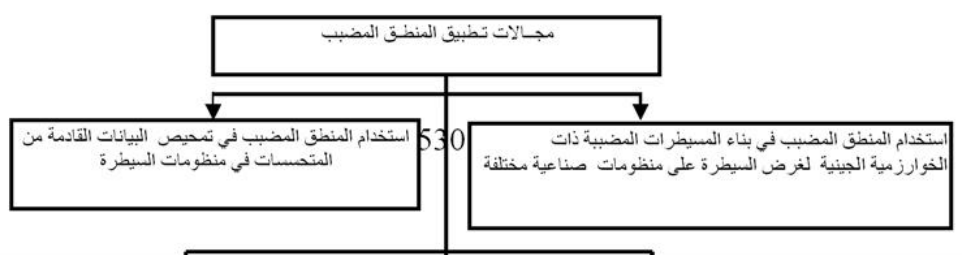


4. Cornelius T. Leondes, 1998, "Fuzzy Logic and Expert Systems Applications".

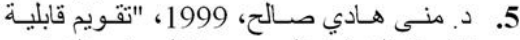

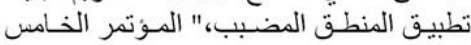

$$
\text { لجامعة بابل. }
$$

6. Kasabov N.K., 2005, "Hybrid Connections Production Systems: An Approach to Releasing Fuzzy Expert Systems," Journal of Systems Engineering for Signal Processing, IEEE Communications Society.

7. Hiroaki Kikuchi, 2006, "Knowledge Acquisition Based on Fuzzy Switching Functions"

8. Mukaidono M., 2006, "Kleene Algebra's in Fuzzy Truth Table Values", the fourth Inter, Workshop on Rough sets, Fuzzy sets, and Machine Discovery University of Tokyo.

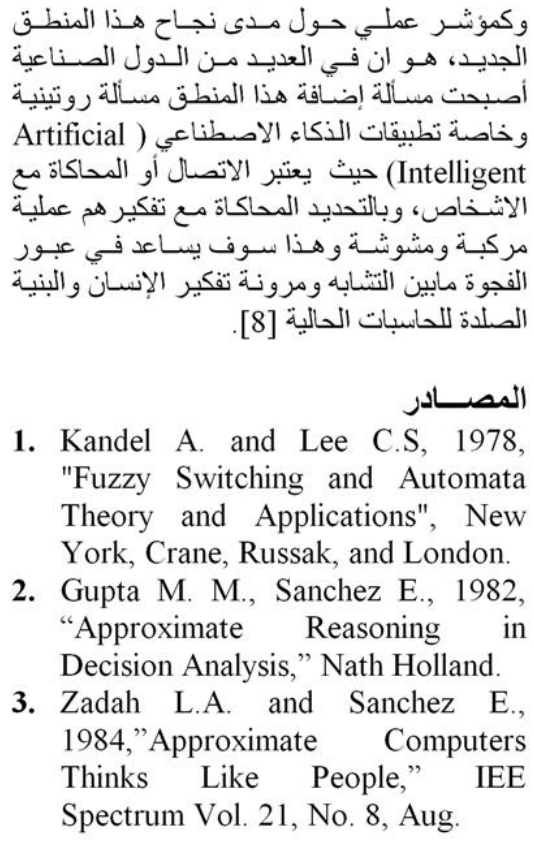




\title{
Study and Analysis the Mathematical Operations of Fuzzy Logic
}

\author{
Muna Hadi Saleh* \\ *Baghdad University/ College of Education Women / Computer Science Department \\ Key words: Fuzzy Set Theory, Full membership, Non-Membership, Contained, \\ Strictly Contained, Absolute Complement, Relative Complement, Union, Intersection, \\ Symmetrical Difference, Boolean Sum, Algebraic Product, Algebraic Sum, Direct \\ Sum.
}

\begin{abstract}
The last decade of this 20th century provides a wide spread of applications of one of the computer techniques, which is called Fuzzy Logic. This technique depends mainly on the fuzzy set theory, which is considered as a general domain with respect to the conventional set theory. This paper presents in initiative the fuzzy sets theory and fuzzy logic as a complete mathematics system. Here it was explained the concept of fuzzy set and defined the operations of fuzzy logic. It contains eleven operations beside the other operations which related to fuzzy algebra. Such search is considered as an enhancement for supporting the others waiting search activities in this field.
\end{abstract}

